\title{
Supporting Information for Interfacial Properties of Water on Hydrogenated and Fluorinated Graphene Surfaces: Parametrization of Nonbonded Interactions
}

\author{
Amir Taqieddin, Mohammad Heiranian and Narayana R. Aluru* \\ Department of Mechanical Science and Engineering \\ Beckman Institute for Advanced Science and Technology \\ University of Illinois at Urbana-Champaign, Urbana, Illinois 61801, USA
}

* Corresponding Author, e-mail: aluru@ illinois.edu, web: http://aluru.mechse.illinois.edu/, phone: +1 (217) 333-1180.

This Supporting Information file includes two data files, two figures, and two tables. We provide the optimized structure coordinates of the hydrogenated and fluorinated graphene as Supporting Information Note 1 and 2, respectively. The structure files are provided in VASP format.

In Supporting Information Note 3, we provide the convergence figure for the RPA method. Figure S1 shows the convergence of $\Delta \mathrm{E}_{\mathrm{exx}}$ and $\Delta \mathrm{E}_{\mathrm{c}}$ as a function of the supercell size, $\mathrm{N}_{\mathrm{s}}$, and the number of k-mesh, $\mathrm{N}_{\mathrm{k}}$.

Finally, Supporting Information Note 4 shows a summary table of the measured and computed contact angle of water data that is used in Figure 2c. Supporting Information Note 5 shows a figure of the computed contact angle using CHARMM FF and a table of the CHARMM FF parameters. 
Supporting Information Note 1. VASP POSCAR of the optimized hydrogenated graphene supercell.

\begin{tabular}{|c|c|c|}
\hline \multicolumn{3}{|l|}{ H_C_cell } \\
\hline \multicolumn{3}{|l|}{1.0} \\
\hline 5.0466456413 & 0.0000000000 & 0.0000000000 \\
\hline 2.52332282065 & 4.3705 & 0.0000000000 \\
\hline 0.0000000000 & 0.0000000000 & 40.0000000 \\
\hline \multicolumn{3}{|l|}{$\mathrm{CH}$} \\
\hline \multicolumn{3}{|l|}{88} \\
\hline \multicolumn{3}{|l|}{ Cartesian } \\
\hline 0.061048362 & 0.805114925 & 1.686423540 \\
\hline 1.322742105 & 0.063982263 & 2.142371178 \\
\hline 2.584393978 & 0.805083334 & 1.686259270 \\
\hline 3.846023560 & 0.063934267 & 2.142344475 \\
\hline 0.061043672 & 2.281721830 & 2.142634869 \\
\hline 2.584397316 & 2.281688690 & 2.142473698 \\
\hline 1.322701097 & 3.022841930 & 1.686547995 \\
\hline 3.846062183 & 3.022836685 & 1.686507702 \\
\hline 2.584342241 & 2.280261755 & 3.254970551 \\
\hline 3.845846176 & 0.062856786 & 3.254833221 \\
\hline 0.060986128 & 2.280425787 & 3.255135298 \\
\hline 1.322811723 & 0.062985249 & 3.254864693 \\
\hline 0.061020561 & 0.806360424 & 0.572774410 \\
\hline 2.584321499 & 0.806193173 & 0.572605848 \\
\hline 1.322525978 & 3.023665667 & 0.572902381 \\
\hline 3.846132278 & 3.023609638 & 0.572865427 \\
\hline
\end{tabular}


Supporting Information Note 2. VASP POSCAR of the optimized fluorinated graphene supercell.

\begin{tabular}{|c|c|c|}
\hline \multicolumn{3}{|l|}{ F_C_cell } \\
\hline \multicolumn{3}{|l|}{1.0} \\
\hline 5.1290931702 & 0.0000000000 & 0.0000000000 \\
\hline 2.5645465851 & 4.44193 & 0.0000000000 \\
\hline 0.0000000000 & 0.0000000000 & 40.0000000000 \\
\hline \multicolumn{3}{|l|}{$\mathrm{C} \quad \mathrm{F}$} \\
\hline \multicolumn{3}{|l|}{88} \\
\hline \multicolumn{3}{|l|}{ Cartesian } \\
\hline 0.056220166 & 0.810641646 & 3.115197182 \\
\hline 1.338471651 & 0.058857709 & 3.609974384 \\
\hline 2.620842457 & 0.810653031 & 3.115206957 \\
\hline 3.903036833 & 0.058914136 & 3.609959602 \\
\hline 0.056182299 & 2.312795877 & 3.609772205 \\
\hline 2.620793343 & 2.312782764 & 3.609747410 \\
\hline 1.338525653 & 3.064518452 & 3.115216255 \\
\hline 3.903095484 & 3.064485073 & 3.115179062 \\
\hline 2.621940851 & 2.311362982 & 4.980365276 \\
\hline 3.904177427 & 0.057789568 & 4.980578899 \\
\hline 0.057193805 & 2.311432123 & 4.980385780 \\
\hline 1.339526534 & 0.057611216 & 4.980623245 \\
\hline 0.057941671 & 0.809073031 & 1.744608402 \\
\hline 2.622778416 & 0.809125006 & 1.744617224 \\
\hline 1.340290070 & 3.063261032 & 1.744630575 \\
\hline 3.904931068 & 3.063121319 & 1.744605303 \\
\hline
\end{tabular}




\section{Supporting Information Note 3. Convergence of the RPA energies}
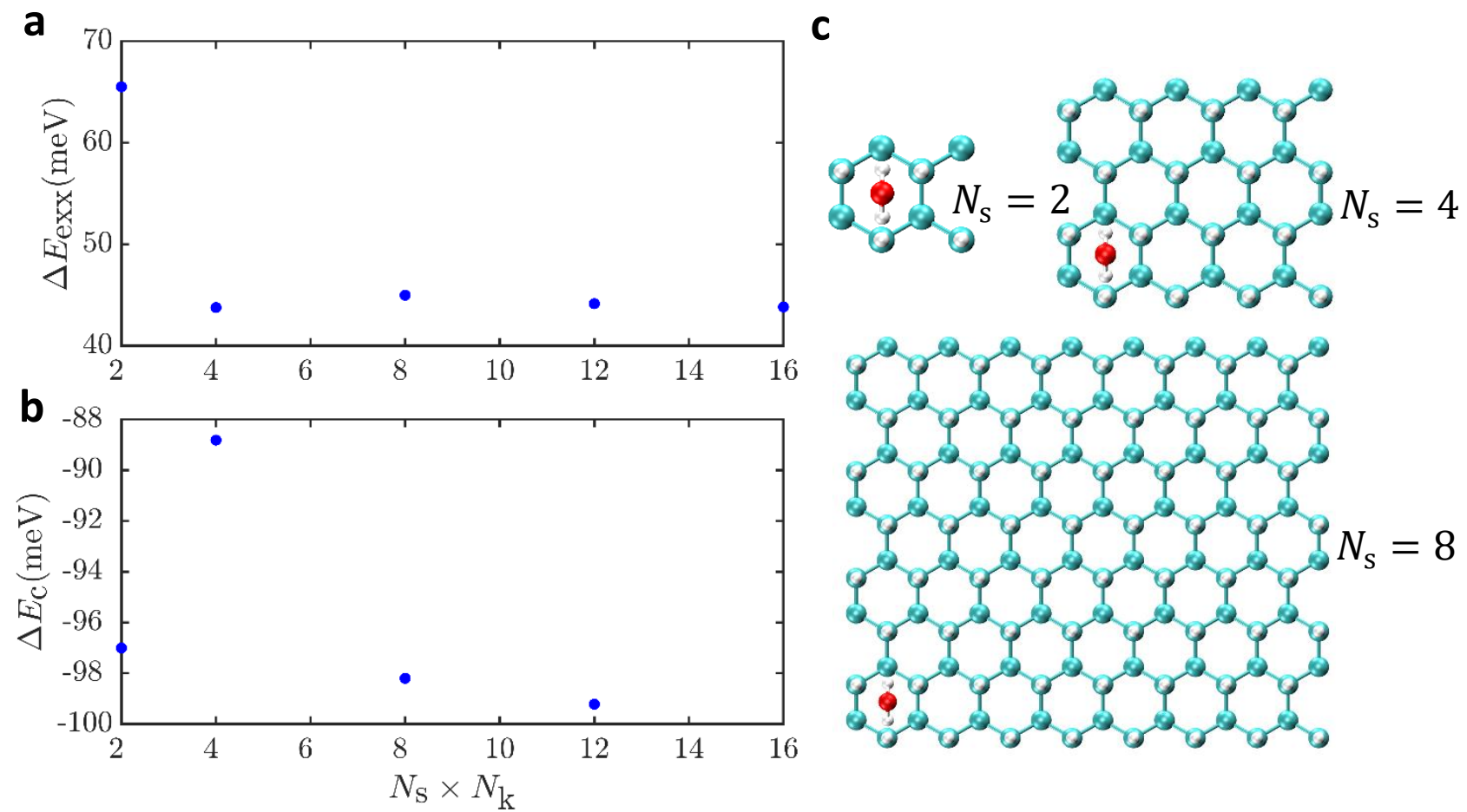

Figure S1. Convergence of the interaction energy between one water molecule and hydrogenated graphene surface for a) the exchange energy, $\Delta E_{\text {exx }}$, and b) the correlation energy, $\Delta E_{\mathrm{c}}$, (oxygen, hydrogen, and carbon atoms are shown in red, white, and cyan, respectively) as a function of different combinations of the $N_{\mathrm{s}} \times N_{\mathrm{s}} \times 1$ supercell size as shown in c), and the $N_{\mathrm{k}} \times N_{\mathrm{k}} \times 1$ Brillouin zone sampling Monkhorst-Pack k-meshes. 


\section{Supporting Information Note 4. Comparison of the contact angle of water}

Table S1. Summary of the measured and computed contact angle of water data used in Figure 2c.

\begin{tabular}{|c|c|c|c|c|}
\hline Surface & Source & $\begin{array}{c}\text { Computational/ } \\
\text { Experiment }\end{array}$ & $\begin{array}{c}\text { Contact angle } \\
\text { (degrees) }\end{array}$ & Condition \\
\hline Pristine & $\operatorname{Ref}^{1}$ & Experiment & $42 \pm 3$ & Free-standing \\
\hline Pristine & $\operatorname{Ref}^{2}$ & Computational & $36.4 \pm 3.5$ & In vacuum \\
\hline $\begin{array}{c}\text { Hydrogenated (Pristine with } \\
\text { H2 Plasma) }\end{array}$ & $\operatorname{Ref}^{1}$ & Experiment & $68 \pm 5$ & $\begin{array}{l}\text { On copper } \\
\text { substrate }\end{array}$ \\
\hline $\begin{array}{c}\text { Hydrogenated (Pristine with } \\
\text { H2 Plasma) }\end{array}$ & $\operatorname{Ref}^{1}$ & Experiment & $41 \pm 7$ & Free-standing \\
\hline $\begin{array}{l}\text { Hydrogenated (hydrogen } \\
\text { atoms are on one side of the } \\
\text { graphene) }\end{array}$ & $\operatorname{Ref}^{3}$ & Experiment & $42 \pm 2$ & $\begin{array}{l}\text { On } \mathrm{SiO} 2 \\
\text { substrate }\end{array}$ \\
\hline Hydrogenated & $\operatorname{Ref}^{4}$ & Computational & $75 \pm 2$ & In vacuum \\
\hline Hydrogenated & This work & Computational & $54.7 \pm 3$ & In vacuum \\
\hline $\begin{array}{l}\text { Fluorinated (fluorine atoms } \\
\text { are on one side of the } \\
\text { graphene) }\end{array}$ & $\operatorname{Ref}^{3}$ & Experiment & $95 \pm 5$ & $\begin{array}{l}\mathrm{On} \mathrm{SiO} 2 \\
\text { substrate }\end{array}$ \\
\hline Fluorinated & $\operatorname{Ref}^{5}$ & Experiment & $\begin{array}{c}104.9-85.9 \\
\text { (depends on } \\
\text { the plasma } \\
\text { exposure time) }\end{array}$ & $\begin{array}{l}\text { On } \mathrm{SiO} 2 / \mathrm{Si} \\
\text { substrate }\end{array}$ \\
\hline Fluorinated & This work & Computational & $73.8 \pm 6$ & In vacuum \\
\hline
\end{tabular}




\section{Supporting Information Note 5. Details of the computed contact angles using CHARMM FF}

Table S2. Summary of the CHARMM FF parameters which are used to compute the contact angles in Figure 2c. The charges of $\mathrm{C}, \mathrm{H}$, and $\mathrm{F}$ are set equal to the obtained charges in our RPA approach. The interactions between the SPC/E water and $\mathrm{C}, \mathrm{H}$, and $\mathrm{F}$ atoms are computed using the mixing rule.

\begin{tabular}{|c|c|c|c|}
\hline Surface & Interaction & $\sigma(\AA)$ & $\varepsilon(\mathrm{kcal} / \mathrm{mol})$ \\
\hline \multirow{2}{*}{ HGR } & C-C & 0.032 & 4 \\
\cline { 2 - 4 } & H-H & 0.045 & 2.68 \\
\hline \multirow{2}{*}{ FGR } & C-C & 0.06 & 3.8 \\
\cline { 2 - 4 } & F-F & 0.135 & 3.26 \\
\hline
\end{tabular}
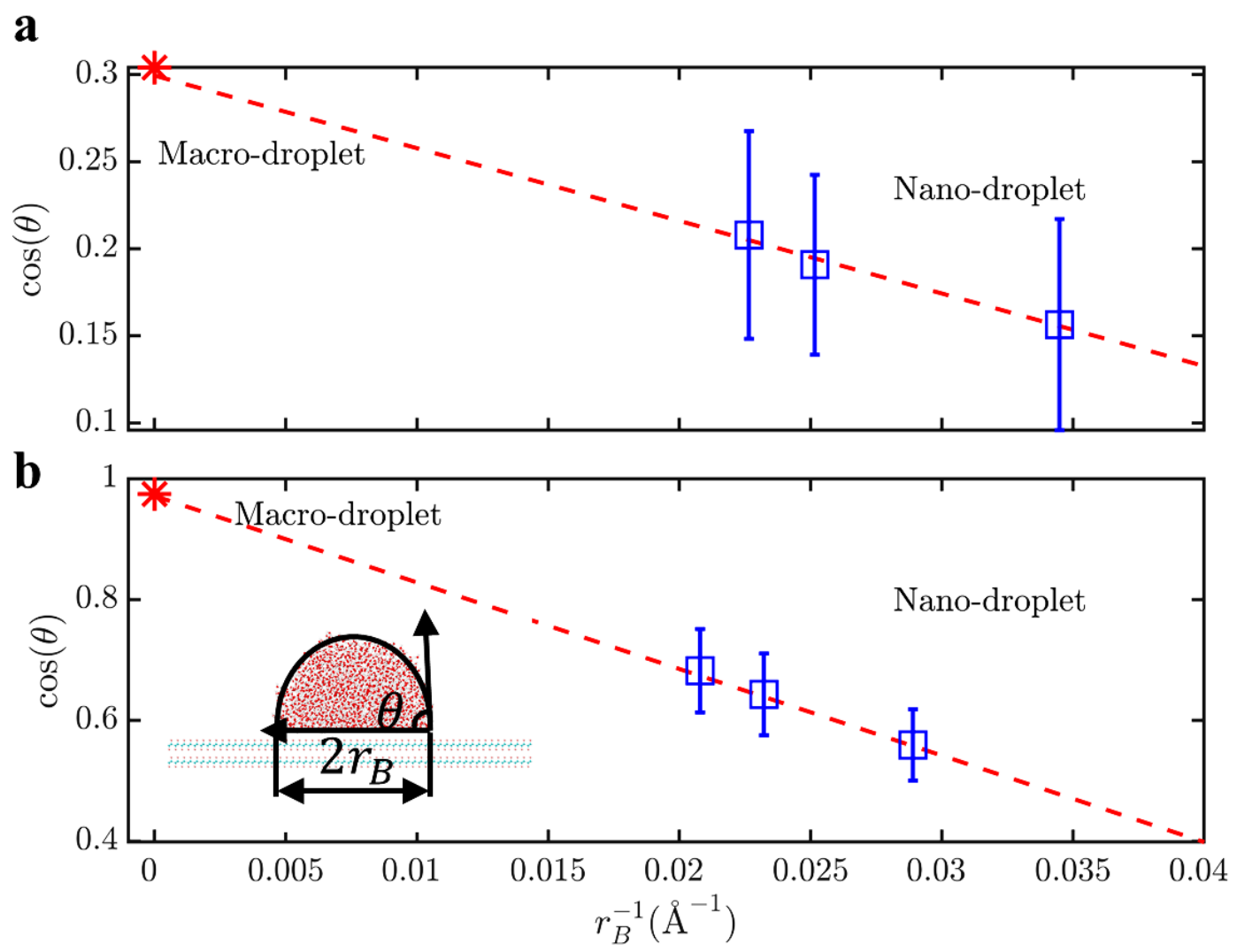

Figure S2. a-b) Water contact angles on two layers of clean functionalized graphene surface for a) hydrogenated graphene, and b) fluorinated graphene where the angles in the nano-droplet region (blue symbols) are computed using the CHARMM parameters in Table S2 and the macro-droplet angle (red curve and the symbol) is extrapolated using the Young's equation. 


\section{References}

1. Prydatko, A. V.; Belyaeva, L. A.; Jiang, L.; Lima, L. M. C.; Schneider, G. F., Contact angle measurement of free-standing square-millimeter single-layer graphene. Nat. Commun. 2018, $9(1), 4185$.

2. Wu, Y.; Aluru, N. R., Graphitic carbon-water nonbonded interaction parameters. J. Phys. Chem. B 2013, 117 (29), 8802-13.

3. Son, J.; Buzov, N.; Chen, S.; Sung, D.; Ryu, H.; Kwon, J.; Kim, S.; Namiki, S.; Xu, J.; Hong, S.; et al. Tailoring surface properties via functionalized hydrofluorinated graphene compounds. Adv. Mater. 2019, 31 (39), e1903424.

4. Vanzo, D.; Bratko, D.; Luzar, A., Wettability of pristine and alkyl-functionalized graphane. J. Chem. Phys. 2012, 137 (3), 034707.

5. Lim, T.; Ju, S., Control of graphene surface wettability by using CF 4 plasma. Surf. Coat. Technol. 2017, 328, 89-93. 